Volume 10 Issue 4

October 2019

\title{
Negotiation, Reciprocity, and Reality: The Experience of Collaboration in a Community- Based Primary Health Care (CBPHC) Program of Research with Eight Manitoba First Nations
}

Wanda Phillips-Beck

First Nation Health and Social Secretariat of Manitoba, wphillips-beck@fnhssm.com

Grace Kyoon-Achan

University of Manitoba, grace.kyoonachan@umanitoba.ca

Josée G. Lavoie

University of Manitoba, josee.Lavoie@umanitoba.ca

Nicholas Krueger

University of Manitoba, umkruegn@myumanitoba.ca

Kathi Avery Kinew

First Nation Health and Social Secretariat of Manitoba, kakinew@fnhssm.com

Stephanie Sinclair

First Nation Health and Social Secretariat of Manitoba, ssinclair@fnhssm.com

Naser Ibrahim

University of Manitoba, naser.Ibrahim@umanitoba.ca

Alan Katz

University of Manitoba, Alan_Katz@cpe.umanitoba.ca

Recommended Citation

Phillips-Beck, W., Kyoon-Achan, G., Lavoie, J. G., Krueger, N., Kinew, K. A., Sinclair, S., Ibrahim, N., \& Katz, A. (2019).

Negotiation, reciprocity, and reality: The experience of collaboration in a community-based primary health care (CBPHC)

program of research with eight Manitoba First Nations. The International Indigenous Policy Journal, 10(4).

doi: https://10.18584/iipj.2019.10.4.8334 


\title{
Negotiation, Reciprocity, and Reality: The Experience of Collaboration in a Community-Based Primary Health Care (CBPHC) Program of Research with Eight Manitoba First Nations
}

\begin{abstract}
This article shares experiences and lessons learned through a collaboration between the University of Manitoba, the First Nation Health and Social Secretariat of Manitoba (FNHSSM), and eight First Nation communities in Manitoba. We employed a participatory approach from planning the research project, to data collection, and to the analysis, interpretation, and implementation of results. We learned that successful collaborations require: a) investing time and resources into developing respectful research relationships; b) strong leadership and governance; c) clearly defined roles and responsibilities; d) meaningful participation of First Nations; e) multiple opportunities for community engagement; and f) commitment to multiple, ongoing, and consistent forms of communication. All factors are integral to creating and maintaining the integrity of the research collaboration.
\end{abstract}

Keywords

First Nations, Indigenous research, research, collaboration, participatory research, research partnerships

\section{Acknowledgments}

We acknowledge the eight participating First Nations in Manitoba: Fisher River Cree Nation, Pinaymootang, Birdtail Sioux, Ebb and Flow, Northlands Denesuline, Cross Lake Band, Berens River, and Nisichawayasik.

\section{Creative Commons License (c) (1) (1)}

This work is licensed under a Creative Commons Attribution-Noncommercial-No Derivative Works 4.0 License. 


\section{Negotiation, Reciprocity, and Reality: The Experience of Collaboration in a Community-Based Primary Health Care (CBPHC) Program of Research with Eight Manitoba First Nations}

In this article, we describe the process by which a university-community partnership evolved with eight First Nation ${ }^{1}$ communities and a cadre of community and university-based researchers in Manitoba. We share what we have learned through our experience of negotiating and pioneering an innovative research collaboration built on a foundation of trust, respectful relationships, meaningful participation, and community engagement. It has been generally noted in the literature that building relationships is necessary for developing research partnerships (Baydala, Saylor, \& Ruttan, 2013; Baydala, Worrell, et al., 2013; Christopher, Watts, McCormick, \& Young, 2008). Yet, few studies detail the process by which these relationships are created. It is our intention to contribute an understanding of this process to the literature: the process by which meaningful relationships are built and collaborations are successfully formed with First Nation communities in Canada.

The backdrop for this article is a program of research entitled Innovation in Community-Based Primary Health Care (CBPHC) Supporting Transformation in the Health of First Nation and Rural/Remote Communities in Manitoba (iPHIT). This study is a 5-year research collaboration between researchers from the University of Manitoba, eight Manitoba First Nation communities, and the First Nations Health and Social Secretariat of Manitoba (FNHSSM), also recognized by the traditional name Nanaadawewigamig, which means "A Healing Place" in the Anishinaabe language. FNHSSM was established in 2013 by the Assembly of Manitoba Chiefs (AMC) Chiefs-in-Assembly, the political organization representing 63 First Nations in Manitoba. The goal of FNHSSM is to create a unified health system with, and for, First Nations in Manitoba through research, policy analysis, and advocacy. It is also the home of the Manitoba First Nations Health Information Research Governance Committee (MFN HIRGC), an ethics review body for all research involving First Nation peoples in Manitoba. The MFN HIRGC reviews and approves research proposals to ensure that all research involving First Nation peoples meet four stipulated guiding principles: a) free, prior, and informed consent, both at the individual and collective level; b) First Nations $\mathrm{OCAP}^{\circledR}$ principles that establish First Nation ownership, control, access, and possession of their data; c) respect for First Nation ethical standards; and d) research must be of benefit to First Nations. These principles are essential for the renewal of First Nation self-determination in health and governance. ${ }^{2}$

\section{Context}

The iPHIT program of study is 1 of 12 teams funded by the Canadian Institutes of Health Research (CIHR) under the Community-Based Primary Health Care (CBPHC) initiative. Lavoie et al. (2010) noted multiple challenges in providing health care in First Nation communities, which highlighted a grave need for CBPHC transformation. Extenuating circumstances include the small size of communities, geographical isolation, and the operation of healthcare services and funding agencies across multiple jurisdictions: federal, provincial, regional health authorities (RHAs), private for-profit

\footnotetext{
${ }^{1}$ In this article, we use the accepted term First Nation, one of the three recognized Indigenous Peoples in Canada (First Nations, Métis, and Inuit), to describe the partner communities that were directly involved in the project. We use the term Indigenous when making broader reference to Indigenous people in Canada or internationally.

${ }^{2}$ The Assembly of Manitoba Chiefs (AMC) established MFN HIRGC in 1996. It operates to ensure that research benefits First Nations through guiding principles established by the AMC Chiefs-in-Assembly.
} 
organizations, and self-governing First Nation communities. Health care delivery is further compounded by a complex multi-jurisdictional system for CBPHC funding and delivery in First Nation communities, particularly when the various jurisdictions fail to clearly define their specific responsibilities and neglect to address the impacts of colonization and the residential school system on the health of these communities (Lavoie et al., 2010). It is no surprise that Lavoie et al. (2010) found First Nation communities who exercised greater control over their local health services appeared to be doing better at meeting their community-based health needs. To investigate this phenomenon further, the iPHIT project focused on the experiences and strengths of community-based primary healthcare in First Nation communities, both from a community-based and population health perspective using a holistic and inclusive approach.

The iPHIT team's principal investigators worked together to develop a common vision of the study. Prior to the proposal writing stage, the nominated principal applicant (NPI) invited FNHSSM staff to an initial meeting to discuss the potential research project and to discuss priorities and process. From then on, FNHSSM took on an active role in all decisions related to the design and development of the research project, and each step of the research project was discussed in detail by the university and FNHSSM teams. Decisions were made by keeping the lines of communication open through regular meetings, open dialogue, and by reaching mutual agreement. This created conditions that ensured meaningful engagement of First Nation communities, while respecting the obligations of First Nations organizations and the university.

Academic and First Nation partners agreed upon four goals for the overall iPHIT research project: a) Describe CBPHC service provision in First Nation communities by focusing on the strengths, key factors, and innovations in health care provision that has helped to maintain the wellness of individuals, families, and the community; b) Explore perspectives of First Nations people living on reserve about $\mathrm{CBPHC}$ and why mainstream approaches may be failing; c) Compare the models of governance, community engagement, strengths, and CBPHC service delivery across communities and provide opportunities for communities to learn from each other; and d) Build collaborative relationships with communities and decision-makers to support the implementation of CBPHC innovation to improve health and wellbeing in First Nations in Canada.

The two primary models of care in First Nation communities in Manitoba reflect the type of federal government health funding they receive, which dictates the type of health care delivery available. Nursing stations are generally located in communities that are either isolated or semi-isolated and are mandated to deliver primary health care services, which are for the most part provided by nurses who work in an expanded scope of practice and by visiting physicians. Emergency care is available on a daily basis. The second model of care, found in the majority of First Nation communities, is the health centre or health office. These facilities only deliver essential public health services during regular business hours and are not funded to deliver primary health care or emergency care services on a 24-hour basis. The federal government also funds a variety of community-based health services such as the Native Alcohol and Drug Addiction Program (NAADAP), Home and Community Care (HCC), Brighter Futures, Aboriginal Head Start on reserve, Strengthening Families-Maternal Child Health, and Canada Prenatal Nutrition Program. However, some community-based programs are not funded consistently across communities and are not universally accessible. 


\section{Establishing the Research Partnerships and Methods}

To provide a comprehensive and balanced perspective, multiple methods were chosen and four independent research components emerged: a) A qualitative community-based participatory research project exploring community members' understanding of primary health care and services needed; $b$ ) an administrative data analysis of the rates of hospitalizations for ambulatory care sensitive conditions (ACSC) utilizing the Manitoba Population Research Data Repository housed at the Manitoba Centre for Health Policy (MCHP) within the University of Manitoba; c) Survey methods, looking at patient, provider, and organizational experiences of primary health care services; and finally, d) More in-depth case studies to further examine those communities that excelled in innovation and wellness through the first three projects. We invited eight First Nation communities to participate and all the communities invited agreed. The selection of communities was purposeful, representing different models of service delivery and four of the five linguistic groups in Manitoba: Ojibway, Cree, Dene, and Dakota. The participating First Nations are geographically dispersed throughout Manitoba, four northern and four southern, and ranged from small (a few hundred residents) to large (a few thousand residents) communities. Two communities are isolated (either fly-in or winter ice roads), two semi-isolated (road access but are far from larger centres), and four of the eight are rural communities with all season road access. Half of the communities, all located in southern regions of the province, have health centers, while four of the communities have nursing stations and are in the North. All receive federal funding for health care. All communities contributed some unique perspective or innovation to CBPHC.

Once selected and invitations accepted, health directors in each community approached band leadership and obtained community consent by way of a band council resolution (BCR). A member of the research team was asked to make a presentation to leadership in two of the communities, while the other health directors were provided with information and a summary of the research outlining the potential benefits. The BCRs where subsequently submitted along with the research ethics applications, which received approvals at various intervals in the first year for a total of five sub-projects within the program of research. The study received approval from the University of Manitoba Health Research Ethics Board, the Health Information Privacy Committee (HIPC) of Manitoba, and the MFN HIRGC in the fall of 2013.

Following ethics approvals, a transfer of funding agreement was signed between the University of Manitoba and FNHSSM, who then assumed management and coordination of the program of research. Research agreements outlining tasks and responsibilities of all parties were also signed by the individual First Nation communities. This level of community and First Nation involvement in research is relatively new and represents a significant change from the troubled past of research involving Indigenous Peoples (Schnarch, First Nations Centre, \& National Aboriginal Health Organization, 2004). Historically, research undertaken by institutions and researchers had little or no benefit to Indigenous Peoples or their communities (Campbell, 2014; Schnarch et al., 2004), was undertaken without their knowledge or consent (Moodie, 2010), and/or was unlawful because it violated their human rights. In some cases, information and Indigenous knowledges were extracted, documented, or disseminated with very little input from the community. This type of research has come to be known as "helicopter research" and "data mining" (Campbell, 2014; Moodie, 2010; Schnarch et al., 2004; Smith, 1999). It is no surprise then that Indigenous communities resisted research and researchers based on well-founded distrust due to these past experiences. Fortunately, community-based participatory 
research approaches that emphasize community involvement are being encouraged and used. The iPHIT program of research unfolded amidst this growing awareness and recognition of the fundamental rights of Indigenous people to determine the direction of research. This new approach to research with Indigenous Peoples also requires researchers to not only to act respectfully and ethically, but legally as well (Moodie, 2010).

Over the past two decades, the advocacy of First Nation leaders and the MFN HIRGC and like bodies in Canada and internationally provided the impetus for the movement to develop research in partnership with First Nations and other Indigenous communities (Boffa, King, McMullin, \& Long, 2011). It is also reflected in the Tri-Councip Policy Statement: Ethical Conduct for Research Involving Humans (TCPS2), which provides specific ethical guidelines for research involving First Nations, Inuit, and Métis in Canada. The Tri-Council Statement emphasizes the importance of collaborating with and engaging Indigenous Peoples and organizations in research (Natural Sciences and Engineering Research Council of Canada [NSERC], the Canadian Institutes of Health Research [CIHR], \& the Social Sciences and Humanities Research Council of Canada [SSHRC], 2014).

In fact, the past several decades have seen Indigenous communities assert their right to not only be engaged in research, but to actually benefit from it and have more control over how research is conducted, including determining the methods that are used (Denzin, Lincoln, \& Smith, 2008). This has prompted health researchers, institutions, and research ethics boards to pay attention to the formation of meaningful research partnerships and to the use and benefits of community-based participatory research (NSERC, CIHR, \& SSHRC, 2014). Community-based participatory research shares characteristics that are compatible with Indigenous methods and often make use of community advisory committees that can function as an anchoring site for building trust, reciprocal relationships, collaborative decision-making, co-learning, and co-creation of knowledge (Gokiert, Willows, Georgis, \& Stringer, 2017). This project came at an ideal time: There was growing recognition that research with Indigenous people must be done differently and mounting pressure to explore alternative research governance models. As a First Nation organization, FNHSSM was well positioned to partner on this project because individuals within the organization had the experience, knowledge, and skills to manage and conduct research respectfully and, more importantly, had existing relationships with First Nation communities. Both the university-based and Indigenous researchers were willing to explore all options, take a leap of faith, and test out an innovative shared-governance model, thus creating a new research landscape in Manitoba. This began with building and bridging those foundational research relationships, and thereby creating lasting relationships that extend beyond the traditional roles of researcher and researched.

\section{Relationship Building}

While paying attention to the formation of meaningful partnerships is relatively easy, actually building and maintaining the relationships that create meaningful partnerships requires concerted effort (KyoonAchan, Lavoie, et al., 2018). Boffa et al. (2011) noted that relationships are necessary at every juncture of the research collaboration including recruiting Indigenous researchers, communicating directly with First Nations Chiefs and Councils, obtaining Indigenous organizational and community support, and

\footnotetext{
${ }^{3}$ The Tri-Council is made up of three federal agencies, representing the natural sciences and engineering, the health sciences, and the social sciences and humanities, with the mandate of funding and supporting research in Canada.
} 
bringing together Indigenous stakeholders with government (provincial and federal) program planners to work with university-based researchers. Others have acknowledged that relationship building requires additional time and energy (Abbott, Dave, Gordon, \& Reath, 2014; Panagiotopoulos, Rozmus, Gagnon, \& Macnab, 2007; Pyett, Waples-Crowe, \& van der Sterren, 2009), but is absolutely critical for establishing trust and confidence in the project and for sustaining partnerships in the long term (Panagiotopoulos et al., 2007). Building this trust is also important on a deeper level for developing a higher level of cultural safety for the research participants over time (Christopher et al., 2008). Successful relationship building facilitates a sense of community ownership and control, along with longterm social environmental policy change or improvements (Rowley et al., 2000). The process by which a community initiates, develops, and implements an intervention program is critical to achieving improved health outcomes (Rowley et al., 2000).

Further highlighting the need for building relationships with Indigenous people, Indigenous scholars have called for frameworks that employ Indigenous methodologies that incorporate tribal knowledge and allow for meaningful representation in research (Kovach, 2009). Kovach (2009) contends that Indigenous frameworks require researchers to "give back" to communities in a manner that is useful. This begins first with identifying which research is relevant and understanding what is useful. This whole process is not possible without first developing respectful relationships and engaging Indigenous communities in the researcher preparation stage (Kovach, 2009).

\section{Project Outcomes}

\section{Why Collaborate with Indigenous Populations in Health Research?}

Immediate positive outcomes are not always the primary goal in collaborating with Indigenous communities, as so often the exchange of knowledge and practice is a valuable outcome of communitybased research itself (Esler, 2008). However, there was a clear intent on the part of the research team to provide partner communities with findings that could be used to support and enhance innovative solutions to the challenges they face in supporting the health of their populations. We learned early in the research process that community leaders were able to use the information they collected to make immediate improvements or address priority concerns that were within their power to do so. For example, after a community results and feedback session in the first year of the research program, one of our partner communities received confirmation that the community's priority was to address the mental health needs in their community and proceeded to work on a strategy to train more than 20 employees in applied counselling. Part of this strategy was to frame mental health within their own definition of primary healthcare and address it as such. After hearing a presentation on their results, another community leader voiced that he now had the validation he needed to move forward will a full language emersion program in their school, as the results had indicated that language and identity were key to community wellness. Both examples have demonstrated that without any further assistance from the research team, benefits were obtained by just hearing the information or having the data to support moving forward on their own priorities. As one health director indicated, "This research and the data had been most valuable in helping us to take our own initiative and look for resources to do what we needed to do" (HD, Community B). 
Not only does collaborating and sharing knowledge give voice to the diversity of community views, but it also ensures that research that is planned and carried out in an ethical and culturally appropriate way (Pyett, Waples-Crowe, \& van der Sterren, 2009). This was exemplified in the research collaboration: Project Elders were present at all yearly gatherings and included in all community workshops ensuring that community specific protocols were followed, and their perspectives were included in interpreting the findings. The findings of the research are the subject of other iPHIT publications (Kyoon-Achan, Lavoie et al., 2018; Kyoon-Achan, Philips-Beck, et al., 2018). It is important to appreciate that the participatory nature of the project resulted in more meaningful and useful data, increased trust in the research process and in the researchers themselves and the benefits of collaborating with First Nation and Indigenous organizations. It is in this respect that we share what we have learned about the process doing research in a good way in partnership with Manitoba First Nation communities over our 5-year journey.

\section{Lessons Learned}

We have distilled our key lessons into six elements of a successful collaboration. These are listed here and discussed in greater detail in the pages that follow.

a. Investing adequate time and resources (funding for on-site visits, hiring community-based researchers, continuous and ongoing communication) is essential for developing respectful research relationships with Indigenous communities;

b. Strong leadership and sound governance are required throughout the research project;

c. Roles and responsibilities need to be clearly defined at the onset of the project, but should be flexible and fluid enough to allow the project to evolve and grow over time;

d. Full and active participation of Indigenous people, communities, and/or organizations, which includes coordination of the project and data management, allowing for approaches and protocols that extend beyond university settings and constraints;

e. Flexibility is critical when engaging Indigenous communities and individuals, as Indigenous communities and organizations have the right to determine their own scope and level of engagement; and

f. Ongoing, consistent, and multiple forms of communication are integral to creating and maintaining the integrity of the research collaboration.

\section{a. Developing the Relationship}

The collaborative work between FNHSSM and university-based researchers (i.e., the iPHIT team, to which eight First Nation communities were later added) began with a brief conversation between the university-based NPI and the research initiatives manager at FNHSSM. Both agreed that the CIHR call for research in community-based primary health care would be of interest and relevance for First Nations in Manitoba. The NPI initiated building the relationship, which began a full year prior to the proposal's due date. Initial conversations developed into a series of discussions and teleconferences 
between the university and FNHSSM-based researchers. Both parties planned and mapped out the research project prior to notification of successful funding. It was agreed that FNHSSM would take an active role in coordinating the research project through a nurse research manager, which is not usual practice and required trust on the part of the university-based researchers. This arrangement resulted in the annual transfer of grant funding to FNHSSM to cover associated costs. We found that the actual transfer of funds was one task accomplished rather seamlessly once the governance model and the underlying principle were understood and accepted by the host university. This decision aligned well with the position of the MFN HIRGC that First Nations be active participants in research, and it made good sense, as FNHSSM had well-established relationships and communication networks with First Nations in Manitoba. The FNHSSM had a wealth of staff who possessed the necessary contextual knowledge and lived experience in working directly with First Nation communities and, as an organization, had a vested interest in health system improvement for First Nations in Manitoba. Several meetings took place to refine the proposal and address how First Nation communities would be engaged and involved for the duration of the research project. While awaiting the outcome of the funding application, FNHSSM drafted a job description, identified office space, sought out interested First Nations, and was fully prepared to hire the nurse research manager to commence working directly with the communities. Upon receiving notification of funding in the summer of 2013, an agreement was signed between FNHSSM and the University of Manitoba to transfer a significant portion of the funding to FNHSSM to hire a nurse research manager, coordinate the project, and work directly with the First Nation communities to hire their own local research assistants. Both the FNHSSM and the university agreed that a nurse with combined experience working in primary healthcare in First Nation communities and in research was necessary to be successful in coordinating the project. The nurse research manager immediately visited the First Nations selected to participate in the study to introduce the project and secure the necessary agreements.

The nurse research manager and research initiatives manager at FNHSSM immediately set follow-up meetings with First Nations health directors and leaders in each of the eight participating communities to discuss what the project entailed and to begin to develop a level of trust that would allow the project to move forward. A few communities required more than one visit by FNHSSM to come to a common understanding and develop community-specific protocols to guide how they wanted to be engaged. These visits often involved making presentations to health staff and leaders in the community, such as Chief and Council or Elders, and were important for ascertaining or verifying community protocols. These meetings were also instrumental for negotiating resources and finalizing arrangements for the supervision and hiring of locally based research assistants (LRAs) and honoraria for Elders who were willing to share their teachings. The meetings were also used to negotiate resources to arrange feasts to start the research off in a good way and, as research data became available, to gather community members to discuss and validate research findings. These provisions often fell within expenditures allowed by the funding agency, but beyond what the university would allow within their own specific policies. This issue was resolved through the research agreement between the FNHSSM and the University of Manitoba, which included the transfer of financial resources to the FNHSSM to coordinate the research project. FNHSSM had more flexibility to cover honorariums and expenditures such as feasts that were directly related to the research and respectful of the communities' cultural protocols and practices. 
Community consent was obtained through a band council resolution (BCR) signed by Chief and Council indicating approval for the health department to participate in the research project. Many phone calls and emails took place between FNHSSM and the leadership in the communities during the drafting of the community research agreements, which in the end were called Statements of Participation (SOP). Positioning the project coordination outside of the university within an organization with staff who were predominantly First Nations was perceived to help with establishing trust, elevating the credibility of the research project, and in bridging relationships and facilitating trust between First Nation communities and the university-based researchers. It also ensured that First Nations ethical protocols were followed. As one Elder commented at the 2015 annual gathering, "Research used to be a dirty word (but) with our own people heading up the research it is no longer that way, they [FNHSSM and university-based researchers] know our ways and know how to do things the proper way. It is important for our ways to be acknowledged and respected such as the giving of sacred medicine-the tobacco" (Elder, KT Workshop, 2015).

\section{b. Strong Leadership and Sound Governance}

The structure of the research project, as shown in Figure 1, allowed the relationships and partnerships to form and to continuously evolve. A research advisory committee was formed consisting of Indigenous leaders, experts in primary health care, and key decision makers from Health Canada and the Government of Manitoba who added high-level insights into the project design, implementation, and its implications. Four independent research sub-projects were developed at the start of the project. By the end of Year 3, a fifth project had been added. Four of the five independent sub-projects were led by a university-based researcher and an Indigenous partner with expertise in the subject area. These project co-leads set out an initial plan that included methods, analysis goals, and target data collection completion dates, and they were responsible for the overall completion of the sub-project. Participation by iPHIT team members varied in the sub-projects and depended on the specific project focus, except for the nurse research manager who participated in all groups and documented their progress. As multiple complex projects often occurred concurrently, coordination of the research involved juggling priorities, reviewing policies, and managing agendas of the iPHIT team's partners. This required strong leadership and organizational skills, and flexibility, both within FNHSSM and the university, in order to operationalize the research plan, as agreed to by all partners. Community-based leadership was also required for organizing, planning, and implementing the research project in their respective communities, but that was not their only contribution to the research. They contributed knowledge of Indigenous health systems and values, culture, and important contextual information that was reflective of their diverse cultures and community's history. As one health director commented during one of our annual gatherings:

This research was very important for our community, it helped us organize and act on our priorities and did so without telling us what was best for us, we figured it out on our own when we looked at our data. (Health Director, Annual KT Workshop, 2016)

Community leaders contributed ideas that were both innovative and important to them. These leadership roles were sometimes filled by the health directors (HDs) in the community, but more often alternated between the LRAs and other community members who stepped up to the role. As leaders, they organized their own project initiatives, gave direction to the iPHIT research team on their specific 
community priorities, and kept the research team abreast of their specific contextual environment and needs. A sound governance structure, such as the one described in the iPHIT project, along with strong leadership is therefore critically important at all junctures, at all levels, and from all partners for an effective collaboration to unfold. This had the added benefit of blending expertise in the creation of the Indigenous and university-based research teams.

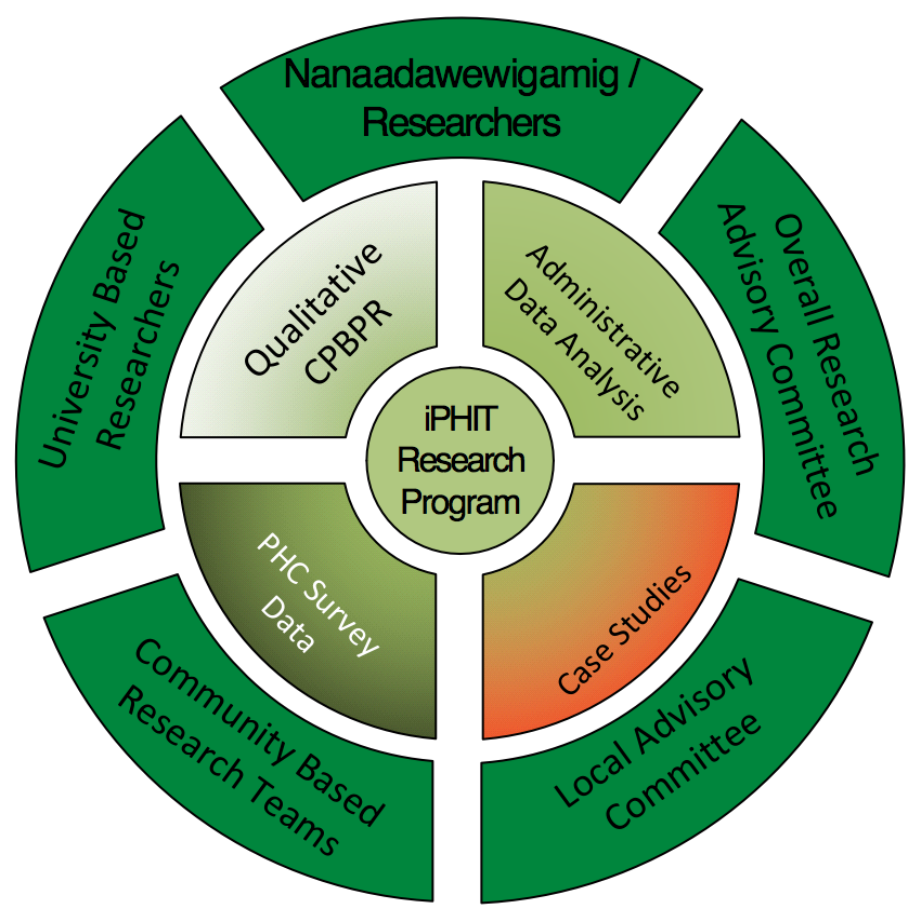

Figure 1. Organization and governance of project. Inner circle: four independent research projects led by university-based and Indigenous researchers. Outer circle: Project partners — university-based researchers, community researchers, local advisory committees, and the overall research advisory team.

\section{c. Clearly Defined, But Fluid Roles and Responsibilities}

The project structure (as shown in Figure 1) and the creation of sub-teams for each study assisted to initially define roles and responsibilities for all partners. Team member roles and responsibilities were negotiated and clearly defined at the beginning of the project with input from all partners so that each unit understood where they fit in the project circle. However, as the project unfolded, roles and responsibilities had some fluidity. For example, leadership at times shifted from one team member to another as skills and experience developed and workloads shifted. In addition, LRAs' and community leaders' roles expanded over time as they gained trust in the process, engaged with the project, generated data, and gained confidence in managing their own community-specific research. Local advisory committees and/or HDs had advisory roles and kept LRAs on track with community priorities, timelines, ethics, and protocols. 
Project roles, although clearly defined at the onset, continued to evolve as some team members stepped back, and others stepped up and took on multiple roles. Each team member's strengths were acknowledged, and they could contribute to the project comfortably over time. The LRAs' primary role was to coordinate and collect data within the community and to help organize community feedback sessions. Later in the research process, they were instrumental in validating and interpreting the results. FNHSSM staff travelled into the communities and functioned as data collectors, when an additional four First Nations and six non-First Nations communities were invited to participate in the survey project. The research advisory committee that had been established at the onset was not involved in the day-today operation of the research beyond the initial kick off meeting in the first months of the project. By Years 2 and 3, community health directors took on the role of overall project advisors. All stakeholders were kept abreast of research activities through bi-annual newsletters and invited to provide input into project direction at yearly gatherings and meetings.

\section{d. Full and Active Participation of First Nation Organizational Partners, Including First Nation Data Management}

FNHSSM was very clear from the initial conversation and planning discussions that they would ensure active participation of First Nations partners in the research. University-based researchers supported and welcomed the level of involvement of FNHSSM and First Nations partners with cautious optimism because a shared governance model had not been tried before on a project of this scale. As a result, FNHSSM not only took part in drafting the research plan, finalizing the proposal, selecting First Nations partners and negotiating arrangements, developing data collection questions and tools, selecting and training LRAs, but also provided overall management of the qualitative data and survey data set.

FNHSSM transferred funding to each participating community to hire the LRAs who were responsible for collecting data. Each community was responsible for recruiting and hiring an LRA and establishing a local advisory committee. Due to their knowledge about their respective communities, LRAs were instrumental in the formation of local advisory teams consisting of Elders, youth, and leaders. These teams were encouraged to meet periodically to provide direction and support to the community-based research project.

LRAs received training from the project nurse research manager and university-based researchers on basic research concepts, interviewing techniques, the use of recording devices, privacy and confidentiality, field management tools, problem-solving capacities, and general data and information management. They participated in drafting, refining, and translating research questions into their respective local languages for respondents who participated using their own languages. The LRAs also provided useful feedback on issues of contextual and cultural appropriateness in the framing of questions and interviewing of respondents. They collected qualitative and survey data through the course of the first 3 years of the project. The LRAs were supported by the nurse research manager and sometimes the research team through weekly or bi-weekly teleconferences and bi-annual visits to the community.

First Nations ownership of data was fundamental for trust to be maintained among iPHIT partners. The one exception was the health administrative dataset, which was housed at MCHP. For this data set, Manitoba Health's Information Privacy Committee requires that all personal health data collected by Manitoba Health and housed at MCHP be analyzed at a secure site at the University of Manitoba. This 
requirement was clearly communicated to and understood by the First Nation communities and by all academic and Indigenous researchers. The analysis of the administrative data was completed by an MCHP-employed analyst bound by the MCHP confidentiality policy. Tables, graphs, and de-identified aggregate data were released to the project researchers and communities for further analysis and interpretation. All other data, such as the interview data, survey data, and focus group data were housed on a secure research server at FNHSSM in accordance with $\mathrm{OCAP}^{\circledR}$ principles. The nurse research manager at FNHSSM coordinated the transcription and management of the data for the project. The study transcripts were returned to the communities to be validated. Each community kept a copy of their raw data in a secure environment within their community.

Community partners were involved in the preliminary analysis of data. They helped clarify words, terms, and concepts emerging from interviews and provided context and explanations of ideas and examples where necessary. As qualitative interviews were completed first, members of that team met to discuss and develop an analysis plan for the data and to decide how best to ensure and maintain community involvement in the study with FNHSSM maintaining coordination of all units and personnel.

\section{e. Acknowledge Multiple Layers and Levels of Community Engagement}

Both Indigenous and university researchers were very aware early on in the research that engaging First Nations may take many forms, which required nuancing the ideal concept of "full and active participation" and acknowledging that not all First Nations communities may participate equally, nor desire to participate equally. The level of participation by each community shifted over time, depending on community priorities and other contextual factors. Understanding this difference was critical for developing effective research partnerships in the iPHIT collaboration. It also respected each community's diversity. Community engagement required initiating contact with First Nation communities early in the process, even prior to developing the final research protocol. In at least one case, it meant multiple face-to-face meetings with community leadership. It also meant attending community feasts, information sessions, making presentations, and being present in the community long before data collection began. Engaging the community was sometimes about building awareness and understanding about the benefits of doing research in the community; other times, it involved making presentations to health staff and community members, answering phone calls, and communicating via email. Most consistent for all communities was the necessity of travelling at a minimum of twice a year to provide information and give research results back to the community. It was also important to acknowledge that First Nation communities vary in their knowledge about research-some have developed their own research priorities, skills, and ethical standards for research engagement and others were just getting started. Most importantly, we learned that meaningful engagement in the research process brings many benefits to both communities and researchers. For example, communities began to take action based on what they learned by participating in data collection and analysis, and researchers were more confident in the data because it went through multiple levels of validation and interpretation by individuals and communities that participated in community and regional forums.

Health directors became increasingly engaged in the interpretation of preliminary results, allowing them to be more aware of the gaps in their health care delivery as well as their rights. They began outlining ways to close the gaps in health service. LRAs also gained confidence in presenting and providing education to communities on the benefits of research. There were also many opportunities for 
researchers to learn from communities, which was reflected in a deeper respect and appreciation for Indigenous and local knowledge. Most importantly, flexibility in allowing communities to decide how and to what extent they wanted to be involved provided an opportunity for them to attend to priorities in their communities, without the pressures of meeting research deadlines or targets. All communities, regardless of the level of involvement, had an opportunity to be co-creators of knowledge, which resulted in more meaningful and applicable information.

\section{f. Ongoing and Consistent Communication is Essential to the Success of the Research Collaboration}

The nurse research manager organized monthly meetings for all four team leads to discuss research progress, provide updates, or analyze data. After the first year of the project, the university team leads' participation waned as other projects, priorities, positions, or suitability changed. She provided frequent updates to team members who took up new roles. Eventually, the study settled into two firm groups, affectionately termed the "qualy" and the "quant" teams. Over the course of the first 3 years, a core group of seven committed university and Indigenous scholars remained and continued to meet monthly. The nurse research manager and NPI met bi-weekly to discuss all project coordination and management. The nurse research manager also organized large team meetings and bought together all academic and Indigenous research team leads, university based-research associates, and local advisory committees from each community once a year to provide updates, make decisions, share results, and network.

The research team learned through this process that communication in many forms and through different media is essential. Face-to-face contact between all partners is necessary to maintain focus and enthusiasm and invest in the research relationship. This requires budgeting sufficient resources for travel to the communities and for community members to travel to meetings and annual gatherings. It also meant having weekly teleconferences and publishing and distributing regular research newsletters and updates. It required adjustment for university-based researchers to trust community-driven processes and/or trust Indigenous and alternative methods as valid and useful. The reverse was also true. Disagreements in perspectives arose periodically on matters of validity or interpretation, but they were all resolved through gathering together and engaging in an open dialogue, which often resulted in coming to some sort of compromise.

\section{Discussion}

The partnership model that evolved through the iPHIT project provides an example of a successful university-community research collaboration and shared governance model. The project focused on the process of establishing relationships with communities, negotiating, and navigating meaningful collaboration between all partners, and keeping two-way lines of communication open throughout the duration of the project (Kyoon-Achan, Lavoie, et al., 2018). The goals of community partners were respected and supported, and communities maintained control over their own community data and research process, which is consistent with seminal work on the subject (Schnarch et al., 2004). Embedding the project in the community, fostering a sense of community ownership and control over the research process, and enabling the community to direct the project could also potentially lead to long-term policy change or improvement (Kyoon-Achan, Lavoie, et al., 2018; Kyoon-Achan, PhilipsBeck, et al., 2018; Rowley et al., 2000). The iPHIT team established a partnership in which there was a 
shared governance structure and methodology that made every attempt to abide by First Nations ethical principles. First Nations were actively involved in decision-making throughout the entire research process from planning, design, data collection, analysis, and the interpretation of data, which included Indigenous methods and relationship building researcher preparation activities as advocated by Indigenous scholars (Kovach, 2009). The November 2010 report on the third wave of the Regional Health Survey makes an important observation: "We [First Nations] recognize that when we have OCAP (ownership, control, access \& possession) of our own data, it returns the power of our own information to our people" (Assembly of Manitoba Chiefs [AMC], 2012, p. 11).

The iPHIT project team can attest that it was successful in returning the power of the data back the First Nations who participated in the research project. This undertaking involved respecting a number of ethical standards. These include the First Nations principles of free, prior, and informed consent, both at the individual and collective levels, and First Nations ethical standards that are in agreement with the requirements of the MFN HIRGC (which became a FNHSSM bylaw). In addition, there are the obligations set out by the Tri-Council for research involving Indigenous Peoples in Canada for community engagement, input, and participation (NSERC, CIHR, \& SSHRC, 2014). In large projects, such as the iPHIT program of research, achieving full control, access, and possession of data may not be possible, or even desirable, but where such arrangements are feasible having an Indigenous organization, such as FNHSSM, house and maintain stewardship over the data goes a long way in building trust and credibility with Indigenous communities, given the long history of mistrust between Indigenous Peoples and research institutions in the past.

Research projects approved by the MFN HIRGC must also be explicit in how they are to benefit the First Nation community as a whole. Achieving this goal requires that researchers initiate research relationships with Indigenous communities or organizations from the very start of the research project, beginning at the idea and proposal writing stage. There must be opportunities for input from Indigenous partners in order to increase the likelihood that the research will be of benefit to and have meaning for them. These recommendations are supported by arguments posited by other research teams (Christopher et al., 2008; Panagiotopoulos et al., 2007). Collaborations that truly engage Indigenous communities by distributing resources and allowing Indigenous organizations, such as FNHSSM, or communities to hire experienced research managers and assistants demonstrate true commitment to meaningful engagement and participation. The unique governance structure of the iPHIT project facilitated the productive discussion and sharing of ideas, bi-directional learning, and balanced perspectives. Engaging the communities in a manner and level that was meaningful to them in the form of local advisory teams, consisting of Elders, youth, and leadership, also facilitated the cross pollination of knowledge, including Indigenous methods of data collection, analysis, and interpretation of the data (Kyoon-Achan, Lavoie, et al., 2018). These collaborations must continue to be supported by funders as new arrangements and partnerships form.

We have developed a model of working together by genuinely listening to each other, which allows us to learn and appreciate what institutional and First Nations guiding principles mean. By including First Nations input at each juncture of the research process, we supported and, in some cases, refined how these guiding principles were applied. Flowing investments directly to First Nation communities to recruit and hire their own research staff further strengthened the relationship with the First Nation communities and was mutually beneficial to all partners. We have demonstrated that it is necessary to 
move beyond principles, such as those recommended by Christopher and colleagues (2008), which focused on building trust through acknowledging personal and institutional histories, understanding the historical context of the research, being present in the community and listening to community members, acknowledging the expertise of all partners, and being upfront about expectations. Successful models for working with Indigenous people and entities also require that academic partners integrate participatory processes that encourage both partners to learn from each other, an essential act in acknowledging and reconciling past abuses inflicted by researchers on tribal communities (Christopher et al., 2008). Our findings are consistent with previous studies demonstrating that relationship building is essential for community collaboration. This approach requires additional time and energy from researchers (Abbott et al., 2014; Panagiotopoulos et al., 2007; Pyett et al., 2009; Sorensen, Fowler, Nash, \& Bacon, 2010), but we found it is absolutely critical for obtaining the consent of community residents, establishing confidence in the findings, and for ongoing opportunities to investigate issues and possible solutions for the health of the community. Collaborations are not collaborations if there is no opportunity to set project priorities jointly; plan, share in decision-making, and discuss research findings; and occasionally re-evaluate the course of action. These opportunities are created; they do not just happen and coming together requires resources. For the iPHIT project and other projects purporting to be a collaborative venture, it is necessary to invest time and resources to support First Nation participation and active involvement. Planning for these opportunities in the proposal writing stage and allocating adequate funds to support these activities must be carried out in the beginning. Despite doing a lot of upfront planning and allocating a budget toward engaging First Nations communities at the planning stages of the project, we did not anticipate the amount of travel that would be required and found that we did not have adequate amounts budgeted to travel and gather as frequently as we would have liked. We also learned from the First Nation communities that maintaining the LRAs for the entire 5 years of the project would have resulted in better participation for the duration of the project. These are critical factors to achieving high rates of participation, confidence in the findings, and trust among Indigenous groups (Kyoon-Achan, Lavoie, et al., 2018). This is where a First Nations organization, such as FNHSSM, can be a huge benefit.

Collaborative research can be a learning process for non-Indigenous researchers working with Indigenous communities. It requires critical reflection on one's beliefs and acknowledgement of the pervasiveness of colonial thought (Isaak, Campeau, Katz, Enns, Elias, \& Sareen, 2010), and the reality of continuing practices and policies of colonization. It has been cautioned that the process of engaging Indigenous communities, if done uncritically in service of ethics guidelines rather than in service of ethical research, can cause harm (Brunger \& Wall, 2016). It could lead to community fatigue, undermining the community's ability to be effectively involved in the research, and restricting the community's ability to have oversight and control over research.

\section{Conclusion}

We have shared sound, but emerging elements of a successful collaboration based on our experience over the course of a 5-year project with eight Manitoba First Nations. This includes pooling strengths from First Nation communities and partner organizations, respecting institutional and community ethics, embracing and collaboratively combating challenges to build respectful, trusting relationships, and creating space for the co-creation of knowledge. We have shared these lessons from our process to assist others in developing strong research collaborations with First Nations organizations and with First 
Nation communities. We must also emphasize, however, that there are individuals with unique strengths that are often key to building necessary partnerships and bridges. It is critical that strong advocates work to advance First Nations self-determination in research. It is also imperative that university-based researchers keep an open mind and a receptive stance to new approaches and arrangements. All partners have to exercise a great deal of respect, patience, and, at times, compromise. Our work contributes to a growing body of literature on developing ethically sound and effective research practices and relationships in community-university collaborative research.

\section{References}

Abbott, P., Dave, D., Gordon, E., \& Reath, J. (2014). What do GPs need to work more effectively with Aboriginal patients? Views of Aboriginal cultural mentors and health workers. Australian Family Physician, 43(1), 58-63.

Assembly of Manitoba Chiefs (AMC). (2012). First Nations Regional Health Survey (RHS) Phase 2 (2008-2010): Manitoba Regional Report. Winnipeg, Canada: Author.

Baydala, L., Saylor, K., \& Ruttan, L. (2013). Meeting standards for community-engaged Aboriginal health research. Paediatrics \& Child Health, 18(1), 8-9.

Baydala, L. T., Worrell, S., Fletcher, F., Letendre, S., Letendre, L., \& Ruttan, L. (2013). "Making a place of respect": Lessons learned in carrying out consent protocol with First Nations Elders. Progress in Community Health Partnerships, 7(2), 135-143.

doi: https://doi.org/10.1353/cpr.2013.0015

Boffa, J., King, M., McMullin, K., \& Long, R. (2011). A process for the inclusion of Aboriginal people in health research: Lessons from the Determinants of TB Transmission Project. Social Science \& Medicine, 72(5), 733-738. doi: https://doi.org/10.1016/j.socscimed.2010.10.033

Brunger, F., \& Wall, D. (2016). "What do they really mean by partnerships?" Questioning the unquestionable good in ethics guidelines promoting community engagement in Indigenous health research. Qualitative Health Research, 26(13), 1862-1877. doi: https://doi.org/10.1177/1049732316649158

Campbell, T. D. (2014). A clash of paradigms? Western and Indigenous views on health research involving Aboriginal Peoples. Nurse Researcher, 21(6), 39-43.

doi: https://doi.org/10.7748/nr.21.6.39.e1253

Christopher, S., Watts, V., McCormick, A. K., \& Young, S. (2008). Building and maintaining trust in a community-based participatory research partnership. American Journal of Public Health, 98(8), 1398-1406. doi: https://doi.org/10.2105/ajph.2007.125757

Denzin, N. K., Lincoln, Y. S., \& Smith, L. T. (2008). Handbook of critical and Indigenous methodologies. Los Angeles: SAGE.

Esler, D. M. (2008). Participatory action research in Indigenous health. Australian Family Physician, $37(6), 457-459$.

Gokiert, R. J., Willows, N. D., Georgis, R., \& Stringer, H. (2017). Wâhkôhtowin: The governance of good community-academic research relationships to improve the health and well-being of 
children in Alexander First Nation. The International Indigenous Policy Journal, $8(2)$. doi: https://doi.org/10.18584/iipj.2017.8.2.8

Isaak, C. A., Campeau, M., Katz, L. Y., Enns, M. W., Elias, B., \& Sareen, J. (2010). Community-based suicide prevention research in remote on-reserve First Nations communities. International Journal of Mental Health and Addiction, 8(2), 258-270. doi: https://doi.org/10.1007/s11469$\underline{009-9250-0}$

Kovach, M. (2009). Indigenous methodologies, characteristics, conversations and contexts. Toronto, ON: University of Toronto Press.

Kyoon-Achan, G., Lavoie, J., Avery Kinew, K., Phillips-Beck, W., Ibrahim, N., Sinclair, S., \& Katz, A. (2018). Innovating for transformation in First Nations health using community-based participatory research. Qualitative Health Research, 28(7), 1036-1049. doi: https://doi.org/10.1177/1049732318756056

Kyoon-Achan, G., Philips-Beck, W., Lavoie, J., Eni, R., Sinclair, S., Avey Kinew, K., .. Katz, A. (2018). Looking back, moving forward: A culture-based framework to promote mental wellbeing in Manitoba First Nations communities. International Journal of Culture and Mental Health, 18(3), 1-14. doi: https://doi.org/10.1080/17542863.2018.1556714

Lavoie, J. G., Forget, E. L., Prakash, T., Dahl, M., Martens, P., \& O'Neil, J. D. (2010). Have investments in on-reserve health services and initiatives promoting community control improved First Nations' health in Manitoba? Social Science \& Medicine, 71(4), 717-724. doi: https://doi.org/10.1016/j.socscimed.2010.04.037

Moodie, S. (2010). Power, rights, respect and data ownership in academic research with Indigenous Peoples. Environmental Research, 110(8), 818-820. doi: https://doi.org/10.1016/ j.envres.2010.08.005

Natural Sciences and Engineering Research Council of Canada (NSERC), Canadian Institutes of Health Research (CIHR), \& Social Sciences and Humanities Research Council of Canada (SSHRC). (2014). TCPS: Ethical conduct for research involving humans. Retrieved from http://www.pre.ethics.gc.ca/pdf/eng/tcps2-2014/tcps_2 final_web.pdf

Panagiotopoulos, C., Rozmus, J., Gagnon, R. E., \& Macnab, A. J. (2007). Diabetes screening of children in a remote First Nations community on the West Coast of Canada: Challenges and solutions. Rural Remote Health, 7(3), 771.

Pyett, P., Waples-Crowe, P., \& van der Sterren, A. (2009). Engaging with Aboriginal communities in an urban context: Some practical suggestions for public health researchers. Australian and New Zealand Journal of Public Health, 33(1), 51-54. doi: https://doi.org/10.1111/j.17536405.2009.00338.x

Rowley, K. G., Daniel, M., Skinner, K., Skinner, M., White, G. A., \& O'Dea, K. (2000). Effectiveness of a community-directed 'healthy lifestyle' program in a remote Australian Aboriginal community. Australian and New Zealand Journal of Public Health, 24(2), 136-144.

Schnarch, B., First Nations Centre, \& National Aboriginal Health Organization. (2004). Ownership, control, access, and possession (OCAP) or self-determination applied to research: A critical 
analysis of contemporary First Nations research and some options for First Nations communities. Journal of Aboriginal Health, 1(1), 80-95.

Smith, L. T. (1999). Decolonizing methodologies: Research and Indigenous Peoples. New York, NY: Zed Books \& University of Otago Press.

Sorensen, R., Fowler, C., Nash, C., \& Bacon, W. (2010). Addressing the gap in Indigenous health: Government intervention or community governance? A qualitative review. Health Sociology Review, 19(1), 20-33. doi: https://doi.org/10.5172/hesr.2010.19.1.020 